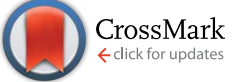

Cite this: RSC Adv., 2016, 6, 94617

Received 6th July 2016

Accepted 27th September 2016

DOI: 10.1039/c6ra17356j

www.rsc.org/advances

\section{Are specific buffer effects the new frontier of Hofmeister phenomena? Insights from lysozyme adsorption on ordered mesoporous silicatt}

\author{
Francesca Cugia, ${ }^{a}$ Silvia Sedda, ${ }^{a}$ Federica Pitzalis, ${ }^{a}$ Drew F. Parsons, ${ }^{b}$ \\ Maura Monduzzi ${ }^{* a}$ and Andrea Salis ${ }^{* a}$
}

\begin{abstract}
Lysozyme adsorption on mesoporous silica at $\mathrm{pH} 7.15$ is buffer specific. The synergistic action of buffers and salts induces relevant effects on the charged interfaces, and thus on lysozyme loading. These findings, rising doubts on the validity of the Henderson-Hasselbalch equation, suggest the occurrence of Hofmeister phenomena also for buffers.
\end{abstract}

Hofmeister (ion specific) effects are phenomena related to the chemical nature of electrolytes. Although they are ubiquitous in all chemical, colloidal, and biological systems,${ }^{1-5}$ they cannot be quantified in terms of the conventional physico-chemical theories (i.e. Debye-Hückel, DLVO, etc.). These are limit theories, based on electrostatics, and valid at infinite dilution only. The gap between theories and Hofmeister related experiments is usually very large since the ion specific effects are generally observed at high concentrations in the presence of strong electrolytes $0.3-3 \mathrm{M}^{6}{ }^{6}$ This is very far from the validity's domain of both limit laws, and their extensions (valid up to $10^{-3}$ to $10^{-2}$ M). ${ }^{3}$ Nevertheless, it has been demonstrated that high concentrations of strong electrolytes are not strictly necessary to observe ion dependent phenomena. Several experiments showed that ion specificity occurs at physiological salt concentrations (0.1-0.15 M), and even below. ${ }^{7,8}$ Very recently, the occurrence of ion specificity at low salt concentrations (Hofmeister charging) has found a theoretical basis. ${ }^{9}$ The finding that Hofmeister effects occur at low salt concentrations poses new questions. Can the weak electrolytes used for buffers give Hofmeister effects? According to the Henderson-Hasselbalch equation, ${ }^{\mathbf{1 0}}$ they should not. In biochemistry, it is

${ }^{a}$ Department of Chemical and Geological Sciences, University of Cagliari-CSGI and CNBS, Cittadella Universitaria, S.S. 554 Bivio Sestu, 09042-Monserrato, CA, Italy. E-mail: asalis@unica.it; monduzzi@unica.it

${ }^{b}$ School of Engineering and Information Technology, Murdoch University, 90 South St, Murdoch, WA 6150, Australia

$\dagger$ Dedicated to Prof. Barry W. Ninham for his $80^{\text {th }}$ birthday.

\$ Electronic supplementary information (ESI) available: Experimental details and additional experimental data. See DOI: 10.1039/c6ra17356j necessary to use a buffer to fix the $\mathrm{pH}$ of the experiment. This procedure has been used in many 'Hofmeister related' studies, in particular on protein aggregation, electrophoretic mobility and enzyme activity. ${ }^{\mathbf{1 1 - 1 5}}$ Typical buffer concentrations are in the range $10-100 \mathrm{mM}$. The implicit assumption is that, due to their low concentration, the buffer ions should not display any specific effect. This is not so. Indeed we recently observed that, even at the same nominal $\mathrm{pH}$, lysozyme electrophoretic mobility was buffer dependent. ${ }^{16}$ The conventional wisdom says that protein charges - and hence electrophoretic mobilities depend on $\mathrm{pH}$ only, regardless of the buffer used to fix it. As shown below, here we confirmed and extended that work. ${ }^{16}$ It should be acknowledged that the original idea to investigate specific buffer effects is due to B. W. Ninham. Following his pioneering work on restriction enzymes, ${ }^{17}$ we then observed specific buffer effects on $\mathrm{pH}$ measurements, ${ }^{18}$ and lipase activities. ${ }^{19}$ Moreover, by looking at the literature more in detail, ${ }^{20}$ we found that similar specific buffer effects were discerned for DNA mobility, ${ }^{21}$ protein stability, ${ }^{22-24}$ antibody aggregation, ${ }^{25,26}$ swelling kinetics of polyelectrolyte gels ${ }^{27}$ etc. Hence, buffers, besides setting $\mathrm{pH}$, may specifically interact with surfaces to modulate their effective charges. ${ }^{20}$ This is not considered in the Henderson-Hasselbalch model for buffer action. ${ }^{28}$

Ordered mesoporous silica (OMS) materials have shown a very high propensity towards the adsorption of proteins and enzymes. ${ }^{29-31}$ The most common OMS materials (i.e. MCM-41, SBA-15), besides a very high surface area, have pore sizes in the range 2-10 $\mathrm{nm}$. These features allow for the penetration of proteins inside the inner architecture of the OMS particles. ${ }^{32,33}$ When an enzyme is adsorbed on OMS an immobilised biocatalyst is obtained..$^{34}$ In nanomedicine applications, OMS can be used to adsorb antibodies or other proteins which either act as targeting agents (molecular recognition), or as therapeutics which require a sustained release. ${ }^{35}$ The potentiality of OMSproteins composites has promoted a growing interest in different applied fields. ${ }^{35}$ In these systems protein/silica interfacial interactions play a fundamental role. It is widely acknowledged that the $\mathrm{pH}$ and the ionic strength of the 


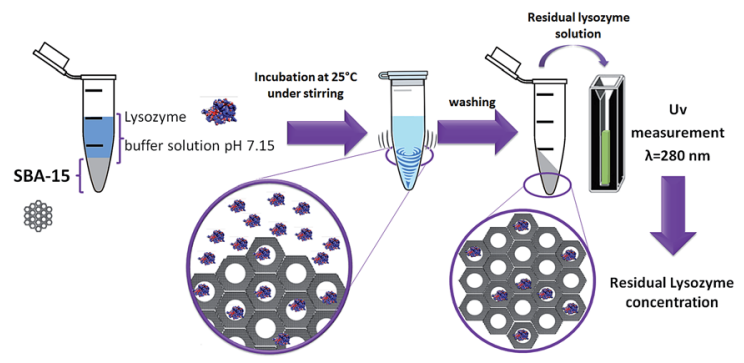

Scheme 1 Schematic representation of the experimental procedure.

adsorbing solution affect the interactions. ${ }^{36-38}$ Less investigated, but not really surprising, is that ions affect specifically protein adsorption on silica. ${ }^{39}$ Generally 'ion-specific' works are carried out by fixing $\mathrm{pH}$ by means of a buffer and then varying the salt type and/or concentration. ${ }^{\mathbf{1 1 - 1 3}}$ Here, our purpose is to demonstrate that, besides strong electrolytes, also weak electrolytes, used to fix $\mathrm{pH}$, play a specific role. This has not yet been investigated systematically. The system chosen to this purpose was the physical immobilisation of lysozyme (LYZ) on SBA-15 and amino functionalised SBA-15 (SBA-NH $\left.\mathrm{NH}_{2}\right)$, which we studied in the presence of different buffers ( $\mathrm{pH}$ 7.15) and different $0.1 \mathrm{M}$ salts. The performed experiments are described in the ESI filet and schematised in Scheme 1.

Briefly, LYZ adsorption on SBA-15 (or SBA- $\mathrm{NH}_{2}$ ) mesoporous silica was carried out at $298 \mathrm{~K}$ and $\mathrm{pH} 7.15$ in the presence of different buffers (Tris, BES, phosphate, and citrate), and quantified through spectrophotometry at $\lambda=280 \mathrm{~nm}$. Buffers dissociation equilibria and $\mathrm{p} K_{\mathrm{a}}$ values are listed in Table 1. Phosphate and citrate are typical buffers occurring in living systems. Tris and BES are commonly used in biochemistry labs. The $\mathrm{p} K_{\mathrm{a}} \mathrm{s}$ values listed in Table 1 , confirm that all buffers would have a good 'buffering action' at a physiological $\mathrm{pH}=7-7.4$. LYZ adsorption was carried out also in the presence of different 100 mM sodium salts, namely: $\mathrm{NaCl}, \mathrm{NaNO}_{3}$ and $\mathrm{NaSCN}$ (and for some experiments also $\mathrm{NaBr}$ and $\mathrm{NaI}$ ). Firstly, the SBA-15 and $\mathrm{SBA}-\mathrm{NH}_{2}$ adsorbent materials were synthesised and characterised according to the procedures reported in the ESI file.t Textural characterisation of SBA-15 (Fig. S1 in ESI provided a surface area (BET) of $777 \mathrm{~m}^{2} \mathrm{~g}^{-1}$, and a maximum of the pore size distribution (BJH) at $6.4 \mathrm{~nm}$ (Table S1 in ESI ). The functionalisation with aminopropyl group to obtain $\mathrm{SBA}-\mathrm{NH}_{2}$ resulted in a decrease of both surface area to $398 \mathrm{~m}^{2} \mathrm{~g}^{-1}$, and pore size to $5.9 \mathrm{~nm}$. The structural characterisation, carried out through SAXS, showed that both SBA-15 and SBA- $\mathrm{NH}_{2}$ have an ordered hexagonal structure with a lattice parameter of $11.7 \mathrm{~nm}$ and $11.6 \mathrm{~nm}$, respectively (Fig. S1C and D, ESI tionalisation with the aminopropyl group produced a change of the sign of the silica surface charge, as demonstrated through potentiometric titrations. The curves of surface charge density $(\sigma)$ as a function of $\mathrm{pH}$ (Fig. S1F, ESI ) (obtained in the absence of buffers or other electrolytes) showed that, at $\mathrm{pH} 7.15, \sigma$ of SBA-15 is negative $\left(-0.04 \mathrm{C} \mathrm{m}^{-2}\right)$, while that of $\mathrm{SBA}^{-\mathrm{NH}_{2}}$ is positive $\left(+0.23 \mathrm{C} \mathrm{m}^{-2}\right)$. This means that, in the absence of any added electrolyte, the two materials have opposite surface charges. Therefore we can expect that the adsorption of LYZ (isoelectric point: $\mathrm{pI}=11$ ) is much more favoured on SBA-15 than on $\mathrm{SBA}-\mathrm{NH}_{2}$.

This is demonstrated in Fig. 1 where LYZ adsorption is reported for the two silica matrices in the presence of different 10 $\mathrm{mM}$ buffers, at the same pH 7.15. In the case of SBA-15 (Fig. 1A) LYZ loading is always higher than $250 \mathrm{mg} \mathrm{g}^{-1}$, and increases along the series Tris $<$ BES $<$ phosphate $<$ citrate. Interestingly, the use of citrate instead of Tris buffer, resulted in a loading increase of $44 \%$. A very low loading (in the range $0-10 \mathrm{mg} \mathrm{g}^{-1}$ ) was instead obtained for $\mathrm{LYZ}$ adsorption on SBA- $\mathrm{NH}_{2}$. This was expected on the basis of electrostatic considerations. For comparison, we also measured LYZ loading by adjusting $\mathrm{pH}$ at about 7.15 with addition of strong acid/base. We obtained a loading of $159 \pm 58 \mathrm{mg} \mathrm{g}^{-1}$ for SBA-15 and about zero for SBA$\mathrm{NH}_{2}$. We assign the high uncertainties to the difficulty to control pH without using a buffer. From the results in Fig. 1 we can argue that the chemical nature of both silica surfaces and buffers is relevant in addressing the observed phenomena. Recent experiments suggested that buffer ions adsorb

Table 1 Buffers used in this work and their $\mathrm{p} K_{\mathrm{a}}$ values at $25^{\circ} \mathrm{C}$. Extracted from ref. 40

Buffer name Dissociation equilibrium

$\mathrm{p} K_{\mathrm{a}}$

Tris [tris(hydroxymethyl) aminomethane]

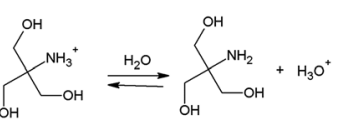

8.06

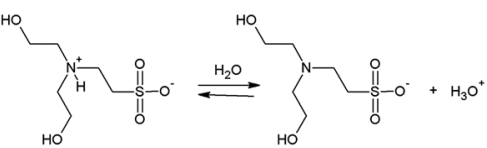

Bes [N,N-bis-(2-hydroxyethyl)-2-aminoethanesulfonic acid]

Phosphate

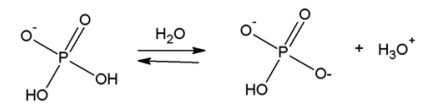

Citrate

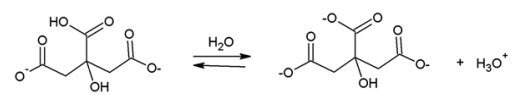




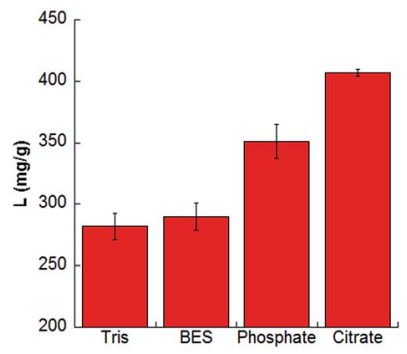

Fig. 1 Effect of $10 \mathrm{mM}$ buffers ( $\mathrm{pH} 7.15 ; T=298 \mathrm{~K}$ ) on $\mathrm{LYZ}$ adsorption on SBA-15 mesoporous silica.

specifically at LYZ surface, thus modifying its effective electric charge, thus affecting its electrophoretic mobility. ${ }^{16}$ Zeta potential $(\zeta)$ measurements shown in Fig. 2 confirm those previous results. Indeed, at $\mathrm{pH} 7.15, \mathrm{LYZ}$ carries a positive net charge thus a positive $\zeta$ value would be expected, independently of the buffer used to fix pH. But Fig. $2 \mathrm{~A}$ shows that the $\zeta$ values depend significantly on the buffer, and decrease along the series Tris $>$ BES $>$ phosphate $>$ citrate. The effect of buffers on $\zeta$ measurements was here investigated also for the silica matrices. In the case of SBA-15 (Fig. 2B) $\zeta$ values are always negative and do not depend on the different type of buffer significantly. On the contrary, in the case of SBA- $\mathrm{NH}_{2}$ (Fig. $2 \mathrm{C}$ ), $\zeta$ values display a clear specific buffer dependence exactly as observed for LYZ (Tris $>$ BES $>$ phosphate $>$ citrate). Remarkably, confirming what previously observed for LYZ, ${ }^{16}$ citrate gives a reversal of zeta potential from positive to negative values also for SBA- $\mathrm{NH}_{2}$. These results demonstrate that the specific effect of buffers is important for LYZ and SBA-NH ${ }_{2}$, but not for SBA-15. Hence, the effect of the buffer (at pH 7.15) can be related to the presence of the positively charged amino groups at the $\mathrm{SBA}-\mathrm{NH}_{2}$ surface, and amino, imidazole and guanidino groups at LYZ surface.

Differently, the negatively charged silanol $\left(\mathrm{SiO}^{-}\right)$groups of SBA-15 are negligibly affected by the different buffers. It is evident that buffers' anions, particularly citrate buffer (constituted by the divalent hydrogen citrate and the trivalent citrate anions, see Table 1) displays a very high affinity and selectivity towards binding at the positively charged interfaces. Hence, a combination of electrostatic, and van der Waals interactions could be suggested to justify the observed trends. ${ }^{36}$ In fact, a more detailed approach has recently been proposed., ${ }^{9,41}$ The effects of increasing the buffers' concentration (from $10 \mathrm{mM}$ to $100 \mathrm{mM}$ ) on LYZ loading on SBA-15 was also studied, as shown in Fig. S2 (ESI:). The increase of the buffers' concentration also affected the absolute value of zeta potentials of SBA-15 (see
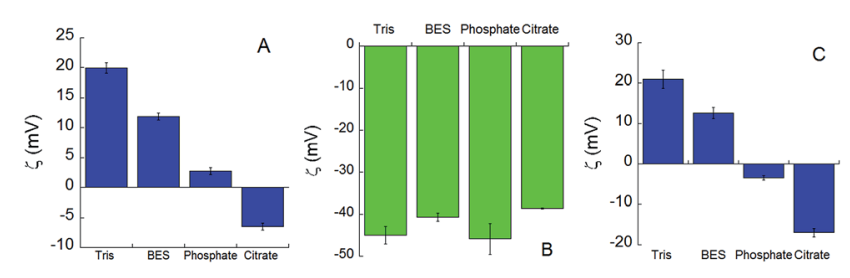

Fig. 2 Specific buffer effects on zeta potential of (A) LYZ (B) SBA-15 (C) SBA- $\mathrm{NH}_{2}(T=298 \mathrm{~K} ; \mathrm{pH}=7.15$; buffer concentration $10 \mathrm{mM})$.

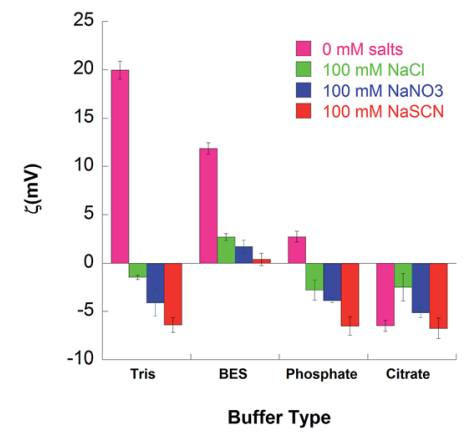

Fig. 3 Effect of strong electrolytes on LYZ zeta potential in different 10 $\mathrm{mM}$ buffers $(\mathrm{pH}=7.15)$.

Fig. S3 ESI:). However, the increase of buffer concentration did not provide any significant advantage to loading, therefore we used a $10 \mathrm{mM}$ buffer concentration to evaluate the effect of adding strong electrolytes.

Different $100 \mathrm{mM}$ sodium salts were added during the LYZ loading experiments on SBA-15 and SBA- $\mathrm{NH}_{2}$. The investigated anions were $\mathrm{Cl}^{-}$and $\mathrm{SCN}^{-}$, that are located respectively in the middle and at the 'chaotropic end' of the Hofmeister series, and $\mathrm{NO}_{3}{ }^{-}$which is in between these two. Fig. 3 shows the effect of the salt addition on $\zeta$-potentials of LYZ in different buffer solutions. The salt addition produces negative $\zeta$ values on LYZ in the presence of all buffers except BES, probably due to the zwitterionic nature of this buffer. Fig. 4A and B show the effect of salt addition on $\zeta$-potential of the two silica matrices in the presence of Tris (cationic) and citrate (anionic) buffers. These buffers were selected since, in the absence of added salt, they gave the lowest and the highest LYZ loading on SBA-15, respectively (Fig. 1). In the case of SBA-15, the addition of salt just decreases the absolute value of $\zeta$-potentials as a result of cations' binding to the negatively charged silica surface.

For SBA- $\mathrm{NH}_{2}$ besides the effect of $\mathrm{NaCl}, \mathrm{NaNO}_{3}$, and $\mathrm{NaSCN}$, we also investigated the effect of $\mathrm{NaBr}$ and $\mathrm{NaI}$, as shown in Fig. 4B. Also in this case the addition of $100 \mathrm{mM}$ sodium salts decreases $\zeta$-potentials, but a 'bell shaped' Hofmeister series is observed (when anions are ordered by the conventional series) in the presence of both buffers. Interestingly, $\mathrm{SBA}-\mathrm{NH}_{2}$ sample is more sensitive than SBA-15 to both buffer and salt type as shown by the corresponding $\zeta$-potentials. Definitely, the addition of salts reduces significantly the surface charge in all cases,
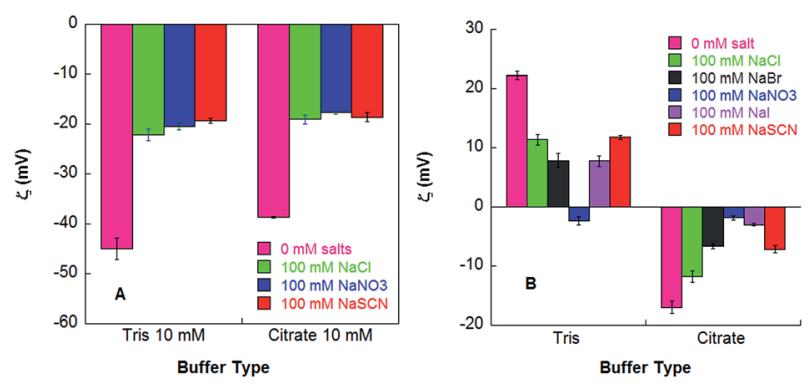

Fig. 4 Effect of salt and buffers on $\zeta$ potentials of (A) SBA-15; (B) SBA$\mathrm{NH}_{2}$. 
but does not cancel the effect of buffers in the cases of the two positively charged surfaces of $\mathrm{LYZ}$ and SBA-NH $\mathrm{N}_{2}$.

Finally, let us focus on LYZ loading in the presence of buffers and salts. Fig. 5 shows the results for the two silica matrices in Tris and citrate buffers: for SBA-NH $\mathrm{N}_{2}$ the effects of $\mathrm{NaBr}$ and $\mathrm{NaI}$ are also enclosed. In the case of SBA-15 (Fig. 5A) in Tris buffer a LYZ loading increase along the series 'No Salt' $<\mathrm{NaCl}<\mathrm{NaBr}$ $<<$ NaSCN was observed. In citrate buffer, instead, ions specificity was lost. Evidently, the background buffer, used to set the $\mathrm{pH}$, triggers a further dependence on the anion type of the added strong electrolytes. In the case of SBA- $\mathrm{NH}_{2}$, LYZ loading data, in the presence of $\mathrm{NaCl}, \mathrm{NaBr}$, and $\mathrm{NaNO}_{3}$, are very low $\left(<20 \mathrm{mg} \mathrm{g}^{-1}\right)$. Strikingly, the addition of either NaI or NaSCN induced a huge increase of loading. This high loading is achieved for both buffers (and also for BES and phosphate buffers: see Fig. S4 in ESI $)$. Fig. 5 shows another noteworthy result: in the presence of $100 \mathrm{mM}$ NaSCN (or NaI), LYZ loading obtained for SBA-15 (Tris buffer) and SBA- $\mathrm{NH}_{2}$ (all buffers) is very similar. In a recent interesting work Meissner $e t$ al. proposed a model for the calculation of the maximum uptake capacity of SBA-15. ${ }^{42}$ A rough comparison between the adsorption data of the present and that work would lead to the conclusion that the very high loading obtained here for SBA- 15 and SBA- $\mathrm{NH}_{2}$ in the presence of $0.1 \mathrm{M}$ NaSCN (or NaI) exceed the maximum loading capacity. In fact, although the Meissner model is highly reasonable, this is based on geometric considerations of pore size and pore volume of the sorbent material as well as LYZ size. There might be several explanations for that apparent inconsistency. In particular, we argue that the different method used to calculate the pore size might be the main cause. ${ }^{43}$ Indeed we used the $\mathrm{BJH}$ method (ESI:), whereas the KJS method was used by Meissner et al. ${ }^{42}$ It has been shown that different methods can result in different pore sizes. ${ }^{44}$ Moreover, we cannot exclude that, in addition to adsorption, an unwanted LYZ co-precipitation phenomenon may occur. A systematic investigation of the reasons of the high loading observed in the presence of $0.1 \mathrm{M}$ $\mathrm{NaI}$ and NaSCN is, although of extreme interest, beyond the scopes of this work which was mainly devoted to investigate the specific effect of buffers.

The high LYZ loading observed for SBA- $\mathrm{NH}_{2}$ found in the presence of the most polarisable anions $\mathrm{I}^{-}$and $\mathrm{SCN}^{-}$, might be due to their ability to counteract, and exceed both the
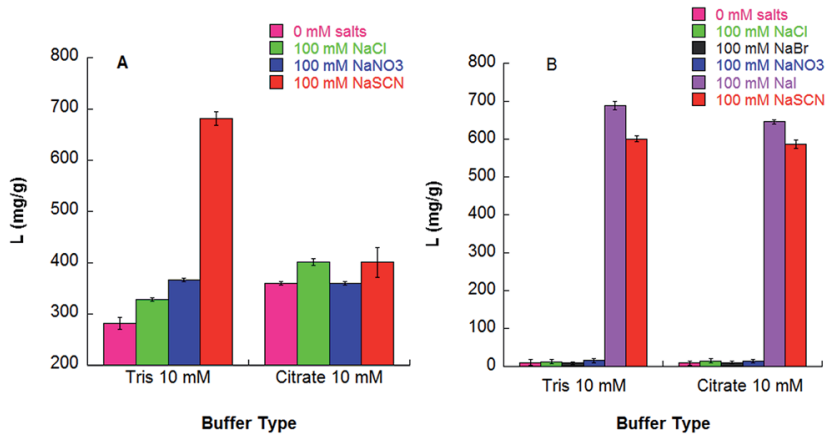

Fig. 5 Effect of buffers $(10 \mathrm{mM})$ and added salts $(100 \mathrm{mM})$ on LYZ loading at $298 \mathrm{~K}$ and $\mathrm{pH} 7.15$ on (A) SBA-15; (B) SBA15- $\mathrm{NH}_{2}$. electrostatic repulsion between the positively charged LYZ and SBA- $\mathrm{NH}_{2}$ surfaces. Definitely, the effect of NaSCN (and NaI) is so strong to overcome all other types of interactions or buffer specific effects involved in LYZ loading. This striking result cannot be justified in terms of electrostatic interactions only, since salt addition reduces significantly all surface charges, as demonstrated by $\zeta$-potential data shown in Fig. 3 and 4 . Indeed, the LYZ loading around $600-700 \mathrm{mg} \mathrm{g}^{-1}$ obtained for both positively and negatively charged silica matrices, is likely promoted by non-electrostatic interactions mainly. A recent work investigating the effect of ionic strength on the adsorption and release of lysozyme from SBA-15 at pH 7 (10 mM phosphate buffer $),{ }^{38}$ showed that a high ionic strength $(1 \mathrm{M} \mathrm{NaCl})$ favours the LYZ adsorption but not its release. Here we fixed pH (7.15) and ionic strength $(100 \mathrm{mM})$ but varied the nature of the buffer used to set $\mathrm{pH}$, the salt used to set ionic strength, and the sorbent surface. The fact that buffers and salt ions give rise to different loadings suggests that they interact specifically with LYZ and silica surface sites, thus affecting their actual dissociation constants and effective surface charges. ${ }^{9,41,45}$ The main outcome is that protein loading on OMS is the result of the complicated interplay among the specific effects of the buffers, the salts, and the charged interfaces. Sometimes the dominant effect is due to the buffer, sometimes to the salt, sometimes both matter. In this work the buffer effect is more important for LYZ loading on SBA-15 (Fig. 1), whereas the specific anion effect is strong also for SBA- $\mathrm{NH}_{2}$ sorbent (Fig. $5 \mathrm{~B}$ ).

Finally, we remark that the consequences of this work go beyond the specific case described here. The Henderson-Hasselbalch equation, known to be one of the pillars of chemistry, considers buffers action only in terms of the $\mathrm{p} K_{\mathrm{a}}$ of the weak electrolyte. But in the presence of charged interfaces the buffer species tend to adsorb thus modifying the effective surface charges and the physico-chemical behaviour of the system. Here, a competition between buffer and salt ions for adsorption on LYZ and OMS surfaces can clearly be inferred. Hence, specific buffer effects would require to be included in the plethora of phenomena that we classify as 'Hofmeister effects'.

\section{Acknowledgements}

Fondazione Banco di Sardegna (PRID 2015) is acknowledged for financial support. F. P. Thanks Agenzia delle Dogane e dei Monopoli for financing her PhD scholarship.

\section{References}

1 P. Lo Nostro and B. W. Ninham, Chem. Rev., 2012, 112, 22862322.

2 F. Tadini-Buoninsegni, M. R. Moncelli, N. Peruzzi, B. W. Ninham, L. Dei and P. Lo Nostro, Sci. Rep., 2015, 5, 14282.

3 A. Salis and B. W. Ninham, Chem. Soc. Rev., 2014, 43, 73587377.

4 C. Carucci, P. Haltenort, M. Salazar, A. Salis and E. Magner, ChemElectroChem, 2015, 2, 659-663. 
5 B. W. Ninham and P. Lo Nostro, Molecular Forces and Self Assembly In Colloid, Nano Sciences and Biology, Cambridge University Press, Cambridge, 2010.

6 A. A. Green, J. Biol. Chem., 1932, 95, 47-66.

7 L. Medda, C. Carucci, D. F. Parsons, B. W. Ninham, M. Monduzzi and A. Salis, Langmuir, 2013, 29, 15350-15358.

8 L. Medda, M. Monduzzi and A. Salis, Chem. Commun., 2015, 51, 6663-6666.

9 D. F. Parsons and A. Salis, Curr. Opin. Colloid Interface Sci., 2016, 23, 41-49.

10 R. de Levie, J. Chem. Educ., 2003, 80, 146.

11 Y. Zhang and P. S. Cremer, Proc. Natl. Acad. Sci. U. S. A., 2009, 106, 15249-15253.

12 A. Salis, F. Cugia, D. F. Parsons, B. W. Ninham and M. Monduzzi, Phys. Chem. Chem. Phys., 2012, 14, 4343-4346.

13 L. Medda, A. Salis and E. Magner, Phys. Chem. Chem. Phys., 2012, 14, 2875-2883.

14 P. Lo Nostro, N. Peruzzi, M. Severi, B. W. Ninham and P. Baglioni, J. Am. Chem. Soc., 2010, 132, 6571-6577.

15 Y. R. Gokarn, R. M. Fesinmeyer, A. Saluja, V. Razinkov, S. F. Chase, T. M. Laue and D. N. Brems, Protein Sci., 2011, 20, 580-587.

16 F. Cugia, M. Monduzzi, B. W. Ninham and A. Salis, RSC Adv., 2013, 3, 5882-5888.

17 H. Kim, E. Tuite, B. Norden and B. W. Ninham, Eur. Phys. J. E, 2001, 4, 411-417.

18 A. Salis, M. C. Pinna, D. Bilanicová, M. Monduzzi, P. Lo Nostro and B. W. Ninham, J. Phys. Chem. B, 2006, 110, 2949-2956.

19 A. Salis, D. Bilanicová, B. W. Ninham and M. Monduzzi, J. Phys. Chem. B, 2007, 111, 1149-1156.

20 A. Salis and M. Monduzzi, Curr. Opin. Colloid Interface Sci., 2016, 23, 1-9.

21 N. C. Stellwagen, A. Bossi, C. Gelfi and P. G. Righetti, Anal. Biochem., 2000, 287, 167-175.

22 M. A. Metrick, J. E. Temple and G. Macdonald, Biophys. Chem., 2013, 184, 29-36.

23 S. O. Ugwu and S. P. Apte, Pharm. Technol., 2004, 86-113.

24 D. S. Katayama, R. Nayar, D. K. Chou, J. J. Valente, J. Cooper, C. S. Henry, D. G. Vander Velde, L. Villarete, C. P. Liu and M. C. Manning, J. Pharm. Sci., 2006, 95, 1212-1226.
25 D. Roberts, R. Keeling, M. Tracka, C. F. van der Walle, S. Uddin, J. Warwicker and R. Curtis, Mol. Pharm., 2015, 12, 179-193.

26 D. Kameoka, E. Masuzaki, T. Ueda and T. Imoto, J. Biochem., 2007, 142, 383-391.

27 L. Y. Chou, H. W. Blanch, J. M. Prausnitz and R. A. Siegel, J. Appl. Polym. Sci., 1992, 45, 1411-1423.

28 H. N. Po and N. M. Senozan, J. Chem. Educ., 2001, 78, 14991503.

29 N. Carlsson, H. Gustafsson, C. Thörn, L. Olsson, K. Holmberg and B. Åkerman, Adv. Colloid Interface Sci., 2014, 205, 339-360.

30 M. Hartmann and X. Kostrov, Chem. Soc. Rev., 2013, 42, 6277-6289.

31 E. Magner, Chem. Soc. Rev., 2013, 42, 6213-6222.

32 M. Piludu, L. Medda, F. Cugia, M. Monduzzi and A. Salis, Langmuir, 2015, 31, 9458-9463.

33 M. Piras, A. Salis, M. Piludu, D. Steri and M. Monduzzi, Chem. Commun., 2011, 47, 7338-7340.

34 J. M. Bolivar, I. Eisl and B. Nidetzky, Catal. Today, 2015, 259, 66-80.

35 A. Baeza, M. Colilla and M. Vallet-Regí, Expert Opin. Drug Delivery, 2015, 12, 319-337.

36 A. Salis, L. Medda, F. Cugia and M. Monduzzi, Colloids Surf., $B, 2016,137$, 77-90.

37 H. Essa, E. Magner, J. Cooney and B. K. Hodnett, J. Mol. Catal. B: Enzym., 2007, 49, 61-68.

38 D. Steri, M. Monduzzi and A. Salis, Microporous Mesoporous Mater., 2013, 170, 164-172.

39 A. Salis, M. S. Bhattacharyya and M. Monduzzi, J. Phys. Chem. $B, 2010,114,7996-8001$.

40 V. S. Stoll and J. S. Blanchard, in Methods in Enzymology, 2009, vol. 463, pp. 43-56.

41 D. F. Parsons and A. Salis, J. Chem. Phys., 2015, 142, 134707.

42 J. Meissner, A. Prause, C. Di Tommaso, B. Bharti and G. H. Findenegg, J. Phys. Chem. C, 2015, 119, 2438-2446.

43 M. A. Smith, M. G. Ilasi and A. Zoelle, J. Phys. Chem. C, 2013, 117, 17493-17502.

44 M. Jaroniec and L. A. Solovyov, Langmuir, 2006, 22, 67576760.

45 C. Bunton, F. Nome, F. H. Quina and L. S. Romsted, Acc. Chem. Res., 1991, 24, 357-364. 